Original Article

Journal of Epilepsy Research pISSN 2233-6249 / eISSN 2233-6257

Received June 10, 2015

Accepted August 9, 2015

Corresponding author: Sandhya Mangalore

Department of Neuroimaging and

Interventional Radiology, National

Institute of Mental Health and

Neurosciences, Bangalore Karnataka,

India

Tel. +08026995424

Fax. +091-080-2656830

E-mail; drsandym@gmail.com

\title{
The Relevance of Interictal Bold Changes to Lateralize Seizure Focus Using Simultaneous EEG-fMRI
}

\author{
Sandhya Mangalore ${ }^{1}$, Rose Dawn Bharath ${ }^{1}$, Neeraj Upadhyay ${ }^{1}$, Ganne Chaitanya ${ }^{2}$, \\ Rajanikanth Panda ${ }^{1}$, AK Gupta ${ }^{1}$, P Satish Chandra', Malla Bhaskar Rao ${ }^{3}$, Anita Mahadevan ${ }^{4}$ \\ Department of ${ }^{1}$ Neuroimaging and Interventional Radiology, ${ }^{2}$ Neurology, ${ }^{3}$ Neurosurgery, and ${ }^{4}$ Neuropathology, \\ National Institute of Mental Health and Neurosciences, Bangalore Karnataka, India
}

\begin{abstract}
Background and Purpose: The main challenge in assessing patients with epilepsy is the localization of neuronal networks involved in seizure generation and the lateralization of seizure onset. Electro encephalogram-functional magnetic resonance imaging (EEG-fMRI) is a noninvasive multimodal imaging technique for epilepsies where the data is acquired based on the inter-ictal epileptiform discharges (IED). Since this is a new technique, the specificity for lateralizing epileptic focus is yet to be established. The peak blood oxygen level dependent (BOLD) signal in an inter-ictal recording is known to correlate with seizure onset focus. In this study we are proposing a simple and practical method without the need for high end post processing techniques of fmri data. The peak BOLD signal derived from EEG-fMRI aids to lateralise seizure focus in a given cerebral lobe (region of interest, ROI). This is a very useful tool in a clinical setting on a given individual clinical case, when other modalities may be conflicting or inconclusive.
\end{abstract}

Methods: We analyzed simultaneous EEG-fMRI of 10 different types of refractory epilepsy. The lateralization index was calculated from the statistical significant clusters obtained between the different ROI and results were validated with other modalities.

Results: Lateralization of seizure focus corroborated well in temporal and extratemporal lobe epilepsy, reflex epilepsy and lesional epilepsy. The only drawback of EEG-fMRI in our study was if insignificant BOLD changes were associated with the given IED.

Conclusions: EEG-fMRI can be helpful additional tool in the pre-surgical work-up of refractory epilepsy particularly when lateralization with other modalities is conflicting or inconclusive. (2015;5:60-69)

Key words: EEG fMRI, Lateralise, Seizure focus, Refractory epilepsy, Interictal discharges

\section{Introduction}

The main aim of evaluation of refractory epilepsy is to identify the epileptic network and lateralize seizure onset, so that surgical interventions can be planned accordingly. Consequentially, the surgery can be planned with increased diagnostic accuracy which will result in a good post operative outcome.

Different modalities capture different components such as neuronal, metabolic or hemodynamic changes associated of the same given physiological phenomenon. Hence to lateralize seizure focus in a clinical setting with multimodal imaging requires a deeper understanding of degree of overlap of neuronal and hemodynamic information.
Electro encephalogram-functional magnetic resonance imaging (EEG-fMRI) is a noninvasive multimodal imaging technique for epilepsies where the data is acquired based on the inter-ictal epileptiform discharges (IED). Since this is a new technique, the specificity for lateralizing epileptic focus is yet to be established.'

Scalp electroencephalogram (EEG) measures the neuronal activity but has limited spatial resolution. ${ }^{2}$ Video electroencephalogram (VEEG) considers the clinical features as well as the concomitant brain electrical activity and helps in identifying the seizure onset but its ability to spatially localize continues to be of a limited value and depends on the expertise of the interpreting neurologist. ${ }^{2}$ Invasive EEG such as subdural EEG includes localized cortical electrical recording based on the suspected seizure focus along with concomitant 
scalp EEG recording from the surrounding areas. Invasive EEG is considered as gold standard with high sensitivity and specificity to detect irritative and epileptogenic zones but it is an invasive technique and is not cost effective to be implemented in all cases. Also subdural EEG has a limited field of view (FOV). Localization with high density EEG and magneto encephalography are reported to have good concordance $^{3,4}$ with the post operative outcome, but spatial resolution is moderately high and is not very sensitive in these modalities.

Dedicated high resolution magnetic resonance imaging (MRI) is useful only when some structural lesion is identified and in such cases the lesion correlates with seizure onset in $80 \%$ of cases. ${ }^{5}$ Singlephoton emission computed tomography (SPECT) and fMRI can still be used in cryptogenic epilepsy cases as it reflects the hemodynamic changes associated with neuronal activity. fMRI has a better resolution of upto $3 \mathrm{~mm}$ as compared to SPECT which has a resolution of $10 \mathrm{~mm}^{6}$

The presurgical evaluation is based on the consensus of the above given techniques in order to overcome the various disadvantages mentioned above. Hence simultaneous multimodal imaging such as EEG-fMRI of inter-ictal epileptiform discharges (IEDs) are thought to provide information about the epileptic networks. The methodology combines the spatial resolution of MRI with the temporal resolution of EEG and its application in pre-surgical evaluation has been an important motivation in developing this technique. ${ }^{7-12}$

A study by Thorton ${ }^{1}$ using EEG-fMRI in focal lesional epilepsy, wherein the onset of IED was convolved into the fMRI has shown the results to be concordant with expected seizure onset defined by electro clinical information in $90 \%$ of the cases.
Many automated methods are being proposed to detect seizure focus, but all these methods have the inherent pitfall of need for extensive post processing of data and this may be cumbersome in regular clinical practice.

We are proposing a simple technique to lateralise seizure focus in different types of refractory epilepsy on a per case basis by using lateralization index (LI) from EEG-fMRI derived blood oxygen level dependent (BOLD) signal. This methodology will aid a diagnosis when other techniques are inconclusive. For purpose of validation we have derived apriori the region of interests (ROls; cerebral lobes) from the other modalities and compared it with the calculated LI. EEG-fMRI is not an all-inclusive entity but it acts as an additional aid to increase the diagnostic confidence in a multimodal epilepsy workup.

\section{Methods}

We recorded simultaneous EEG-fMRI in 20 patients of refractory epilepsy but were able to obtain well formed IED in EEG in 10 patients only.

\section{Clinical details}

We classified the epilepsy based on the ILAE classification (Fig. 1) and included lesional and non-lesional, reflex, temporal and extra temporal lobe refractory epilepsy.

The patients of temporal and extratemporal epilepsy had definite imaging findings and were planned for surgical resection whereas reflex and cryptogenic epilepsy were evaluated with imaging and VEEG as a part of evaluation and were planned for conservative manage-

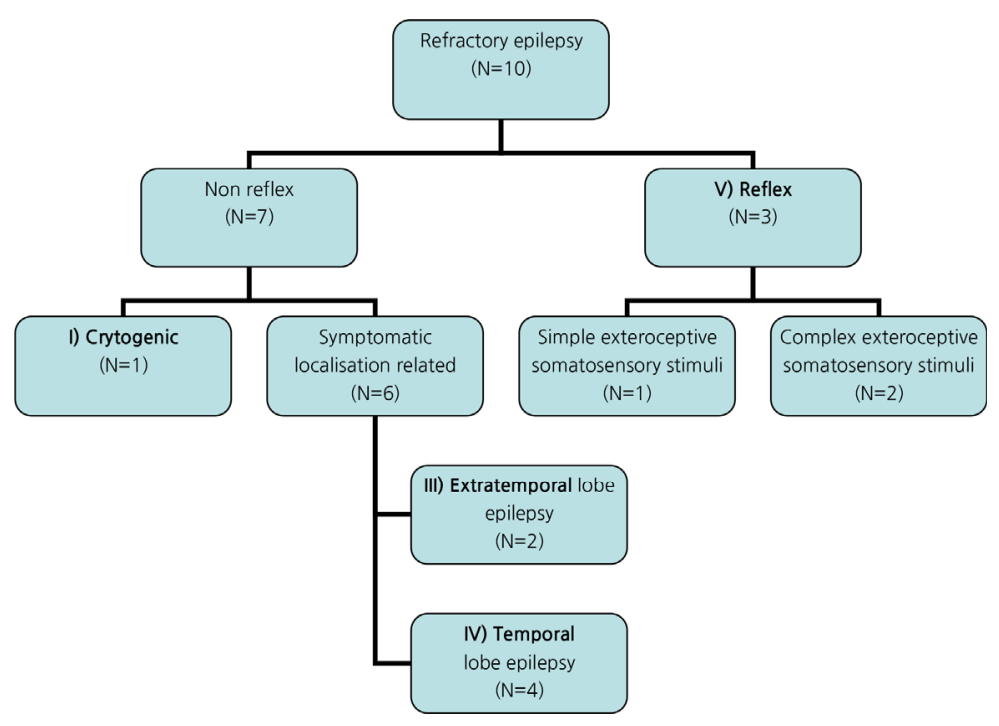

Figure 1. Flow chart. 
ment. The presurgical evaluation to lateralise epilepsy with respect to cortical lobe involved was based on imaging (MRI/SPECT/positron emission tomography [PET]), VEEG and neuropsychology and the decision as to the side of resection was determined using these modalities. Cryptogenic and reflex epilepsy cases were planned for medical management and VEEG and SPECT were the only modalities available to lateralise the seizure focus. EEG- FMRI was performed in order to validate the findings of EEG-fMRI with other standard modalities. All the cases selected were of refractory epilepsy so as to increase the yield of obtaining of IED during EEG-fMRI acquisition. The EEG-fMRI findings were correlated with findings from other modalities and the validation was based on the consensus of the findings of other standard modalities. Subdural recordings were performed in only few cases due to the higher costs involved.

\section{Data acquisition}

A written informed consent was obtained from all the patients (legal guardians in case of pediatric). During the process of the study, they were not subjected to any additional procedures or interventions excepting those dictated by the accepted clinical protocols. 'Trigger stimulus' specific to the type of reflex epilepsy was given to all patients to improve the yield of IEDs and none of the patients had developed overt clinical seizures.

\section{EEG data acquisitions}

EEG was recorded using a 32-channel magnetic resonance (MR) compatible EEG system (Brain Products, Gilching, Germany). MRI compatible electrode cap (BrainCapMR ${ }^{\circledR}$, Germany). The EEG cap consisted of 3 scalp electrodes placed according to the international 10-20 system electrode placement and one additional electrode dedicated to the electrocardiogram (ECG). Data was recorded relative to an $\mathrm{FCZ}$ reference and a ground electrode (10-5 electrode system). ${ }^{13}$ Data were sampled at $5,000 \mathrm{~Hz}$, with a band-pass filter of $0.016-250 \mathrm{~Hz}$ and notch filter at $50 \mathrm{~Hz}$. The impedance level between the electrode and the scalp was kept below $5 \mathrm{k} \Omega$. EEG was recorded using the Brain Recorder software (Version 1.03, Brain Products, Germany). Care was taken to prevent patient movement artifacts. The data was recorded during eyes-closed, relaxed and awake condition. The patient underwent resting state EEG-fMRI sessions each lasting for 9.4 minutes and IEDs were used for the analysis.

\section{fMRI data acquisition}

Resting functional MR-images were acquired using a 3T-scanner (Skyra, Siemens, Erlangen, Germany). Patient's head was positioned within a 20-channel head coil with foam padding to provide comfort and to minimize head movements. The following MR sequences were recorded: a T1 weighted three dimensional high resolution image and echo-planar images (EPI) using BOLD contrast. All axial sections were oriented parallel to the anterior commissure-posterior commissure plane. EPI sequences were obtained 185 volumes were obtained applying the following EPI parameters: $185 \mathrm{EPI}$ volumes were obtained using the following MRI parameters: 34 slices, 6 mm slice thickness without any inter-slice gap, FOV $192 \times 192$ mm, matrix 64 $\times 64,3,000$ ms repetition time, $35 \mathrm{~ms}$ echo time, $90^{\circ}$ refocusing pulse, matrix- $256 \times 256 \times 114$, voxel size- $1 \times 1 \times 1 \mathrm{~mm}$.

\section{SPECT imaging acquisition}

In patients with reflex epilepsy, SPECT was recorded after injecting $20 \pm 2 \mathrm{mCi}$ of meta-stable nuclear isomer of technetium-99 tagged with ethylene cystenate dimer under aseptic precautions. Ictal SPECT was recorded after inducing clinical seizures with specific sensory stimuli. The radiotracer was injected immediately when the patient developed a clinical seizure and after 45 minutes after post injecting the radiotracer, the patient was taken for Ictal SPECT recording. Interictal SPECT was recorded 24 hours later. SPECT images were acquired with SIEMENS SymbiaT6 scanner, (Brain Products GmbH, Germany; low energy, high resolution collimators, matrix resolution: $128 \times$ 128). Iteratively reconstructed brain slices were reoriented based on the orbito-meatal line. SPECT images during inter-ictal phase and ictal phase were analyzed using Lassen's correction software to assess the regional cerebral blood flow (CBF) and areas with highest perfusion during inter-ictal and ictal period were assessed using cerebellum as the reference

\section{EEG-fMRI data analysis}

Steps in the analysis of EEG-fMRI were as follows: a) EEG data artifact removals and preprocessing. b) Onset and duration of each IED after visual inspection of the $\mathrm{EEG}$ signal. c) fMRI data preprocessing. d) EEG information (onset and duration of each IED) convolved into the fMRI data.

\section{EEG data artifact removal and preprocessing}

Raw EEG data was processed offline using Brain Vision Analyzer version 2 (Brain Products, Gilching, Germany). Gradient artifact cor- 


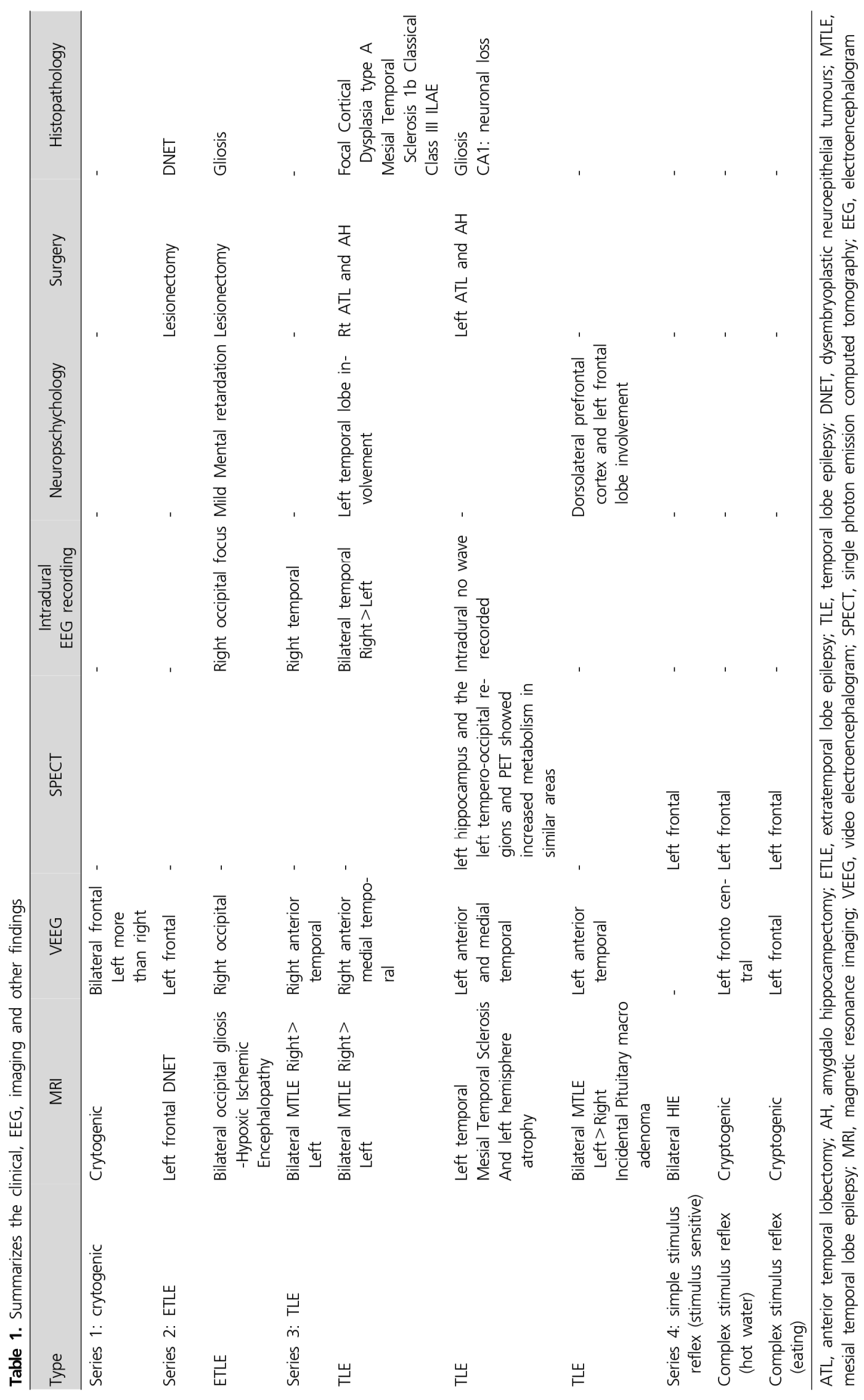




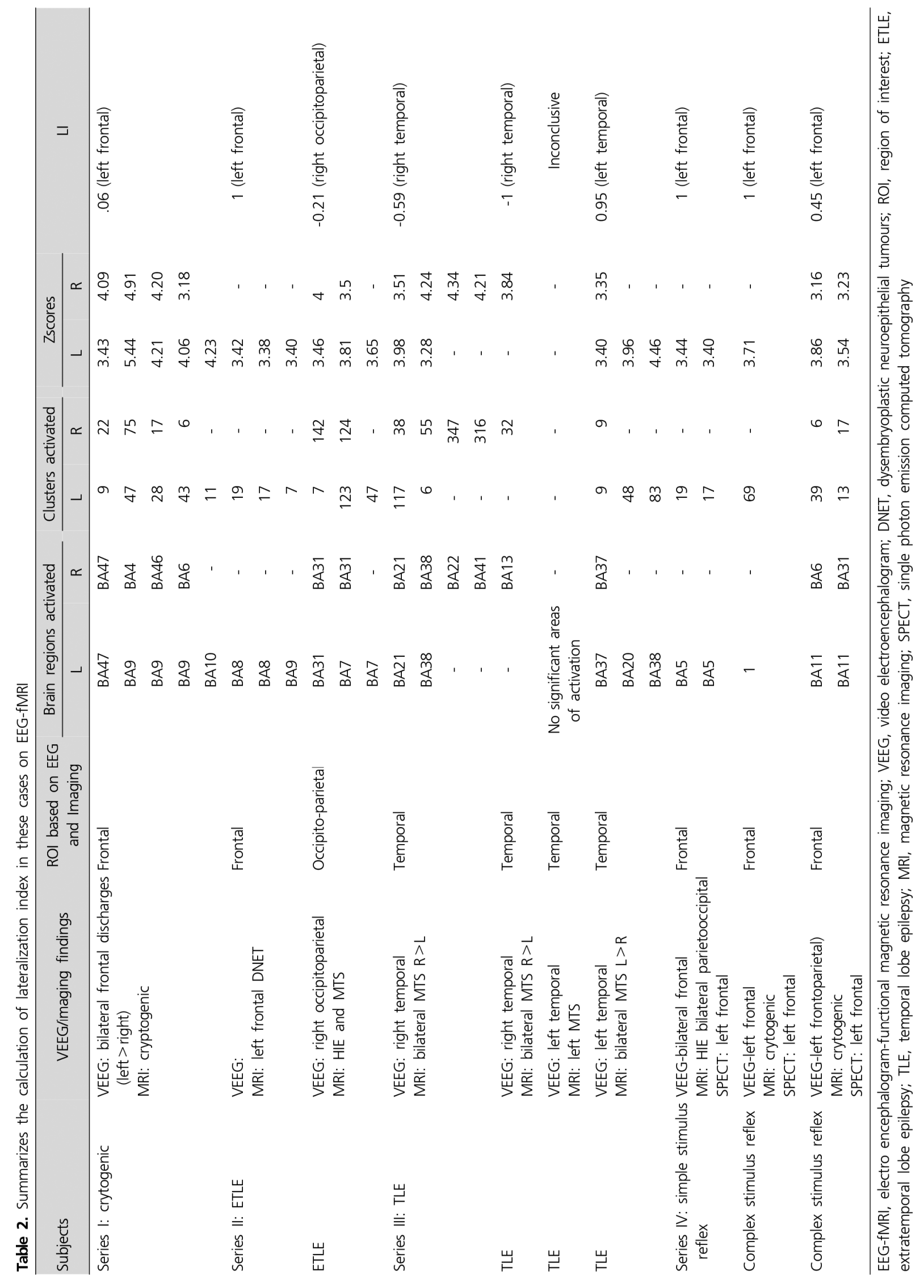


rection was performed using modified versions of the algorithms ${ }^{14}$ where a gradient artifact template is subtracted from the EEG using a baseline corrected sliding average of $20 \mathrm{MR}$-volumes. Data was then down-sampled to $250 \mathrm{~Hz}$ and low-pass filtered with an Infinite Inverse Response filter and a cut-off filter at $70 \mathrm{~Hz}$. Following gradient artifact correction, the data was corrected for cardio ballistic artifacts using an average artifact subtraction method ${ }^{15}$ provided in Brain Vision Analyzer version 2. This method involves subtracting the artifact on a second by second basis using heartbeat events (R peaks) detected in the previous $10 \mathrm{~s}$. It requires accurate detection of $R$ peaks which is aided by the employment of a moving average low pass filter and a finite impulse response high pass filter. ${ }^{15}$ In the present study, the R peaks were detected semi-automatically, with manual adjustment for peaks misidentified by the software. To average the artifacts in the EEG channels, the $R$ peaks were transferred from the ECG to the EEG with a selected time delay. The average artifact was then subtracted from the EEG. Once gradient, cardio ballistic had been removed, the data was then inspected visually for artifacts resulting from movement or any other electro-physiological artifact and any epoch containing a those artifact was rejected.

\section{Onset and duration measurement correspond to epileptogenic} discharge from EEG signature

After removal of artifacts from EEG signal recorded in the MR environment, the EEG was examined for IEDs by visual inspection by an experienced epileptologist. The onset and duration of the IEDs were noted. Statistical parametric maps were generated for each of these sessions correlating seizures to BOLD response.

\section{EEG informed $\mathrm{fMRI}$ analysis}

For the analysis of the resting functional data at the single subject level, we treated the IEDs of EEG as single trial "event related potentials" in an event related design, considering them as explanatory variables in the general linear model (GLM) analysis. However instead of performing a conventional analysis of the data with epileptogenic discharge, we modeled the input function using the onset time, and the duration of each IED and convolved them with a gamma hemodynamic response function, adopting a variable epoch model. ${ }^{16}$ By providing the GLM with the EEG parameters we gain a better fit of the model thus making a more accurate prediction of fluctuations in IEDs.

\section{fMRI analysis}

The fMRI analysis was performed using statistical parametric map- ping (SPM8; Welcome Department of Cognitive Neurology, London, $U K)$. The first five fMRI frames of each time series were discarded to match the EEG and fMRI time series, giving a total of 180 frames for analysis. The fMRI images were realigned for motion correction by registration to the mean image. The images were normalized to the Montreal Neurological Institute (MNI) template. Images were smoothed with a Gaussian kernel of $6 \mathrm{~mm}$. SPM combines the GLM and Gaussian field theory to draw statistical inferences from bold response data regarding deviations from null hypotheses in three dimensional brain space. Preprocessed data were modeled using the onset time and the duration of each EEG IEDs as an regressor, convolved with a canonical hemodynamic response function. ${ }^{17} \mathrm{~F}$-contrast was used to test for BOLD signal changes related to time of onset of the IEDs. The activated brain areas were labeled after transforming the coordinates of MNI template to Talairach coordinates The Brodman areas activated in the predetermined lobe from other studies (frontal temporal occipital parietal) were considered and the number of voxels and intensity of activation (z score) were considered for further analysis (Table 2). Though the fMRI has good spatial resolution at level of sulci and gyri, the results from other modalities are predicted based on the side of the parcellated lobe involved. Hence we have clustered all the ROI in a given lobe and predicted the results.

\section{Lateralization index calculation}

$\mathrm{ROI}$ based on the lobe involved was considered apriori in each of the epilepsy cases from the VEEG and imaging findings. Lateralization index was calculated using the formula: number of voxels activated multiplied by the intensity of activation (z score) in the given ROI by methodology as given below. ${ }^{18}$ The statistics for every activated voxel has been fixed to significance level of $(p<0.001$ uncorrected extent of threshold 5 )

$$
\mathrm{LI}=-\mathrm{f} \cdot \mathrm{QLH}-\mathrm{QRH} / \mathrm{QLH}+\mathrm{QRH}
$$

where QLH and QRH are representative quantities measured by fMRI for the left hemisphere $(\mathrm{LH})$ and right hemisphere $(\mathrm{RH})$ contributions, respectively. The factor $f$ is a scaling factor that defines the range of $\mathrm{LI}$ values (i.e., $\mathrm{LI}$ varies continuously from -f for pure $\mathrm{RH}$ dominance to $+f$ for pure $L H$ dominance). Value of $f$ was held to 1 (i.e., LI varies between - to +1 ). ${ }^{18}$

We have used a statistical threshold of LI compared to a pre-defined threshold (LITH) value at 0.2 . A LI of 0.2 suggest that the cortical volume activated in $\mathrm{LH} \mathrm{ROI}$ is $50 \%$ greater than the RH ROI. The seizure focus is determined according to the following rule. ${ }^{18}$ 
LI > LITH (+0.2), left sided lateralization of seizure focus;

LI<-LITH (-0.2) indicates right hemispheric dominance.

The methodology of applying the formula has been demonstrated in patient 2 of extratemporal lobe epilepsy (ETLE) (Supplementary Table 1).

\section{Results}

We recorded simultaneous EEG-fMRI in 20 patients of refractory epilepsy. Good quality EEG was obtained following pulse and gradient artifact subtraction, allowing identification of IEDs in 10 cases. The other 10 cases were discarded as the IEDs were either not identified on the EEG during the 9 minute recording or due to the presence of artifacts that could not be easily corrected for visual interpretation. The yield was $50 \%$. The results will be discussed based on each category of epilepsy.

\section{Series 1: cryptogenic localization related epilepsy}

The clinical presentation in this patient was complex partial seizures with secondary generalization. No identifiable lesion was detected on the MRI. The routine scalp EEG showed bilateral independent interictal epileptiform discharges which were predominantly noted in the frontal regions. These IEDs were more frequently noticed in the left frontal region compared to the right side. There were also frequently occurring generalized bursts of spike followed by a slow wave discharges at a frequency of 3-4 $\mathrm{Hz}$ with fronto central predominance. EEG-fMRI showed significant clusters in left frontal region.and correlated well with VEEG findings.

\section{Series 2: extra temporal lobe epilepsies}

In the first patient the clinical presentation was refractory frontal lobe seizures. A left frontal dysembryonic neurectodermal tumor was identified on the MRI. Lateralization was to the left frontal lobe on VEEG. Left frontal clusters showed significant activation on EEG-fMRI. The results were concordant in the three modalities and patient underwent lesionectomy.

The second patient was noted to have bilateral occipital gliosis on the MRI, a result of peri-natal hypoxic ischemic encephalopathy. The patient presented with medically refractory complex seizures at a frequency of 3-4 attacks per day prior to the surgery and with significant visual impairment. Though lateralization was difficult based on clinical semiology, the seizure onset was lateralized to the right occipital region in the VEEG. EEG-fMRI to showed lateralization to the right occipital region. The result was concordant on all modalities. The gliotic area in the right was surgically resected on the right side and was performed in the right occipital region. Three months post surgery the patient has presented with only 4 short lasting seizure attacks.

\section{Series 3: temporal lobe epilepsy}

There were four patients with TLE. Three cases showed bilateral Mesial Temporal Sclerosis on MRI. In the first two patients VEEG and IEEG showed lateralization of the predominant seizure focus to the right. In both these patients the EEG-fMRI lateralized the seizure focus to the right temporal region (Fig. 2). The patients underwent right anterior temporal lobe resection with amygdalohippocampectomy.

The third patient had left temporal gliosis with associated left anterior temporal atrophy suggestive of left sided mesial temporal sclerosis (MTS) on MRI. VEEG was localizing the seizure focus to the left anterior and medial temporal region. Ictal SPECT showed perfusion changes in the left hippocampus and the left tempero-occipital regions and PET showed increased metabolism in similar areas. The patient underwent left anterior temporal lobe resection with amygdaIo-hippocampectomy. On EEG-fMRI no significant clusters were noted in the the region of interest that is left temporal region and hence results were inconclusive in this case.

In the fourth patient with bilateral MTS left more than right on MRI and the focus was lateralised more on the left on VEEG. Incidentally a pituitary macroadenoma was also identified on the MRI.The surgical
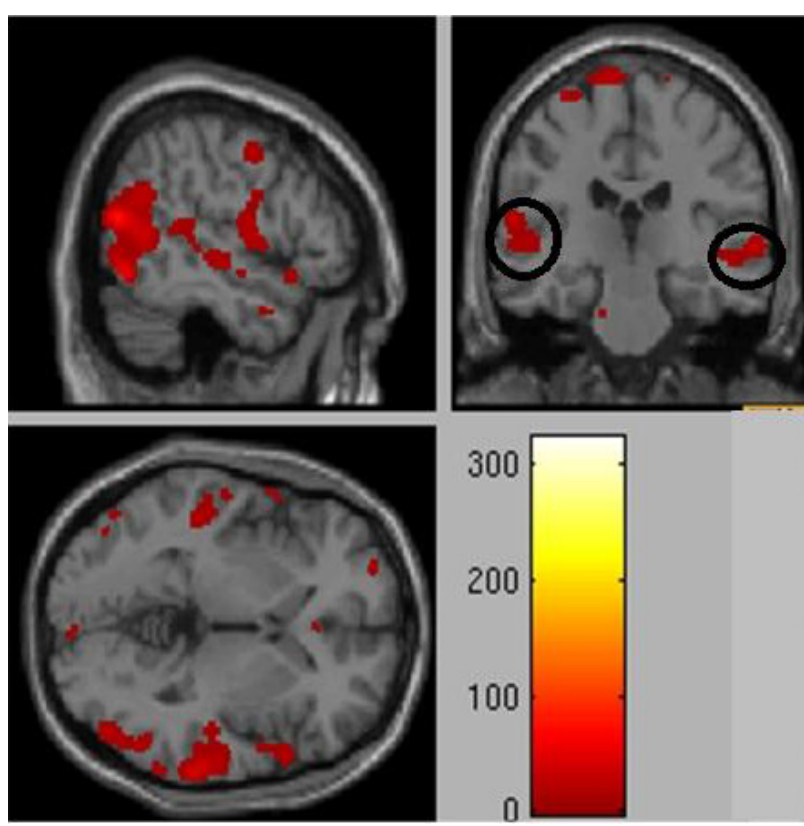

Figure 2. A case of temporal lobe epilepsy with bilateral mesial temporal sclerosis and seizure focus noted in the right temporal lobe. 
decision was deferred and the patient is being further evaluated. EEG-fMRI showed activation in the left superior temporal gyrus.

\section{Series 4: reflex epilepsies}

Three patients were identified in this category of which one of them had simple exteroceptive somatosensory epilepsy (startle epilepsy) while the other two were complex exteroceptive somatosensory epilepsy. In the second group of patients one had eating epilepsy while the other had hot water epilepsy. The patient with the startle myoclonic epilepsy, was found that hypoxic ischemic changes were found predominantly in bilateral parieto-occipital regions. Evaluation with SPECT revealed left frontal lobe involvement. EEGfMRI showed significant activations left fronto-parietal region.

In both the patients with hot water epilepsy and eating epilepsy the MRI was normal. The VEEG was localizing to the left frontal region in the case of eating epilepsy and to the left fronto-central region in the case of hot water epilepsy. SPECT showed focus in left frontal and EEG-fMRI showed significant activations in the left frontal regions.

\section{Discussion}

EEG-fMRI is non-invasive, does not involve the use of radioactive substances and does not require the occurrence of a clinical seizure. It is a technique much less cumbersome when compared to VEEG and subdural EEG and does not carry the risk of exposure to radioactive substances as in SPECT studies. Since EEG-fMRI has high spatial resolution, and unlike other modalities, along with cortical structure it also shows the subcortical structures involved in seizure activity.

Two techniques of modeling IEDs are described. One is based on onset and duration of each IED and other is based on only the onset of each IED. ${ }^{10,19}$ Spike based technique requires large number of spikes (up to 60 spikes) and also the onset segment of IED may have low amplitude potential change and hence the associated BOLD signal may not be significant ${ }^{19}$ and is technically time consuming both to identify these spikes and convolve them in the GLM of fMRI. We have considered convolving onset and duration of each IED in the GLM as it known to have a high degree of sensitivity. ${ }^{10}$ We obtained good BOLD signal changes with a minimum of three IEDs and hence is a good method for analysis.

Studies have shown that areas with maximum BOLD change correlate with seizure onset zone ${ }^{1}$. In our study we considered this max- imum BOLD signal change to correspond to onset zone and used this to lateralize the side of epilepsy. LI was calculated at ROI decided apriori for validation from other modalities such as VEEG, SPECT and sub-dural EEG.

We have demonstrated lateralization in a variety of cases such as Temporal Lobe Epilepsy and Extra Temporal Lobe Epilepsy, Reflex epilepsy, Lesional and Non lesional Epilepsy. In our series of patients with refractory focal epilepsy there was good correspondence between the lateralization of IED related BOLD changes and areas located on VEEG, sub-dural EEG and interictal SPECT.

For the methodology of lateralization index we reviewed papers on the lateralization of language dominance in epilepsy using various paradigms. A review paper on the methodology of calculating $\mathrm{LI}^{18}$ suggested two types that is either whole brain unbiased (global measure) or Region of interest (regional measure) approach. Studies on language lateralization in epilepsy ${ }^{20-23}$ have suggested the ROI method to be the best method.

In our study we have followed the ROI selection method to measure the lateralization index. There are various categories of epilepsy and we have analyzed each case individually as the ROI was different in different cases. Hence we have fixed our statistical threshold at $p$ $<0.001$ uncorrected and extent of threshold 5 to determine BOLD activation in corresponding ROls.

The side with peak BOLD is known to correspond with seizure focus. Hence the lateralization of seizure focus is typically determined by the size of LITH. We have used a statistical threshold of LITH value at $0.2 .^{24,25}$

EEG-fMRI correlated well with other validated standardized techniques such as VEEG, sub-dural EEG and SPECT.

Good lateralization of seizure focus was noted in TLE, ETLE, reflex epilepsy. The only drawback of EEG-fMRI in our study was if insignificant BOLD changes were associated with IED.

Though identifying epileptic zone would be the ultimate aim of any multimodal imaging studies, it requires more meticulous understanding of the physiological changes associated with epilepsy. EEG-fMRI is an analysis based on the inter-ictal activity and how well this translates to identification of seizure onset during ictal discharges and actual electrophysiological activity during an ictus is of concern. Also the gold standard for epileptogenic zone or seizure focus identification is a surgical outcome evaluation, at least one year after surgery. In our study only four patients had surgery and follow-up was not done. Hence epileptogenic zone cannot be defined in this study. However our study provides a methodology of using 
EEG-fMRI to lateralise the side of epilepsy and validates it with other standardised presurgical evaluation tools. A long term followup study using this methodology is warranted.

Significant number of IED is important to get good quality BOLD changes. In our study we recorded EEG-fMRI in 20 cases of epilepsy. Ten cases were rejected as no significant IEDs could be identified and hence the yield like in other studies was $50 \%$ only. ${ }^{19}$

In conclusion, our methodology of using lateralization index is a simple and practical method in a given clinical setting and it correlated well with other validated techniques of identifying seizure focus. Though EEG-fMRI is not an all-inclusive modality, it may aid in decision making in the workup of cases of epilepsy with ambiguous lateralization.

EEG-fMRI as a stand alone technique in work up of clinical cases can only be validated with larger trials.

\section{Acknowledgement}

Cognitive Neuroscience Center is supported by the Department of Science and Technology (DST), India.

Thank our epilepsy team of NIMHANS Sanjib Sinha, P. Satish Chandra from Dept of Neurology, Malla Bhaskara Rao, Arivazhagan from Department of Neurosurgery., Anita Mahadevan from Neuropathology, Shobini Rao, Jamuna Rajeswaran Dept of Neuropsychology.

\section{References}

1. Thornton $R$, Laufs $H$, Rodionov $R$, et al. EEG correlated functional MRI and postoperative outcome in focal epilepsy. Journal of Neurology, Neurosurgery \& Psychiatry 2010;81:922-7.

2. Halchenko YO, Hanson SJ, Pearlmutter BA. Multimodal integration: fMRI, mri, EEG, MEG. Advanced image processing in magnetic resonance imaging 2005:223-65.

3. Knowlton RC, Elgavish R, Howell J, et al. Magnetic source imaging versus intracranial electroencephalogram in epilepsy surgery: a prospective study. Ann Neurol 2006;59:835-42.

4. Knowlton RC, Elgavish RA, Bartolucci $A$, et al. Functional imaging: II. Prediction of epilepsy surgery outcome. Ann Neuro/ 2008;64:35-41.

5. Fujimoto A, Masuda H, Homma J, Sasagawa M, Kameyama S. False Lateralization of Mesial Temporal Lobe Epilepsy by Noninvasive Neurophysiological Examinations-Case Report. Neurologia Medico-chirurgica 2006;46:518-21.

6. Clare S. Functional MRI : Methods and Applications. Degree of Doctor of Philosophy. Nottingham: University of Nottingham. 1997.

7. Lemieux L, Salek-Haddadi A, Josephs O, et al. Event-related fMRI with simultaneous and continuous EEG: description of the method and initial case report. Neuroimage 2001;14:780-7.

8. Krakow K, Woermann F, Symms M, et al. EEG-triggered functional MRI of interictal epileptiform activity in patients with partial seizures. Brain 1999; 122:1679-88.

9. Jacobs J, Boor R, Jansen O, Wolff S, Siniatchkin M, Stephani U. Localization of epileptic foci in children with focal epilepsies using 3-tesla simultaneous EEG-fMRI recordings. Clinical Neurophysiology 2007;118: e50-1.

10. Salek-Haddadi A, Diehl B, Hamandi K, et al. Hemodynamic correlates of epileptiform discharges: an EEG-fMRI study of 63 patients with focal epilepsy. Brain Research 2006;1088:148.

11. Al-Asmi A, Bénar CG, Gross DW, et al. fMRI activation in continuous and spike-triggered EEG-fMRI studies of epileptic spikes. Epilepsia 2003; 44:1328-39.

12. Benson RR, FitzGerald $D B$, LeSueur $L L$, et al. Language dominance determined by whole brain functional MRI in patients with brain lesions. Neurology 1999;52:798-809.

13. Oostenveld R, Praamstra P. The five percent electrode system for high-resolution EEG and ERP measurements. Clinical Neurophysiology 2001;112:713-9.

14. Allen PJ, Josephs 0 , Turner R. A method for removing imagingartifact from continuous EEG recorded during functionalMRI. Neuroimage 2000; 12:230-9.

15. Allen PJ, Polizzi G, Krakow K, Fish DR, Lemieux L.. Identificationof EEG events in the MR scanner: the problem ofpulse artifact and a method for its subtraction. Neuroimage 1998;8:229-39.

16. Warbrick T, Mobascher A, Brinkmeyer J, et al. Single-trial P3 amplitude and latency informed event-related fMRI models yield different BOLD response patterns to a target detection task. Neuroimage 2009; 47:1532-44.

17. Friston KJ, Ashburner JT, Kiebel SJ, Nichols TE, Penny WD. Statistical parametric mapping: the analysis of functional brain images. London: Academic Press, 2007.

18. Seghier ML. Laterality index in functional MRI: methodological issues. Magnetic Resonance Imaging 2008;26:594-601.

19. Grouiller F, Thornton RC, Groening K, et al. With or without spikes: localization of focal epileptic activity by simultaneous electroencephalography and functional magnetic resonance imaging. Brain 2011;134: 2867-86.

20. Billingsley RL, McAndrews MP, Crawley AP, Mikulis DJ. Functional MRI of phonological and semantic processing in temporal lobe epilepsy. Brain 2001;124:1218-27.

21. Baciu M, Watson J, McDermott $K$, et al. Functional MRI reveals an interhemispheric dissociation of frontal and temporal language regions in a patient with focal epilepsy. Epilepsy \& Behavior 2003;4:776-80.

22. Benke T, Köylü B, Visani $P$, et al. Language lateralization in temporal lobe epilepsy: a comparison between fMRI and the Wada Test. Epilepsia 2006;47:1308-19. 
Sandhya Mangalore, et al. Lateralizing seizure focus using EEG-fMRI 69

23. Ries ML, Boop FA, Griebel ML, et al. Functional MRI and Wada determination of language lateralization: a case of crossed dominance. Epilepsia 2004;45:85-9.

24. Pujol J, Deus J, Losilla JM, Capdevila A. Cerebral lateralization of lan- guage in normal left-handed people studied by functional MRI. Neurology 1999;52:1038-43.

25. Binder J, Frost J, Hammeke T, et al. Human temporal lobe activation by speech and nonspeech sounds. Cerebral Cortex 2000;10:512-28. 